\title{
Erinnerung an Paul Bert (1833-1886) und die Entwicklung der Höhenphysiologie in der Schweiz
}

Gerhard Rudolph

\section{SUMMARY}

It is the merit of Paul Bert to have assigned as an important task to experimental physiology the investigation of mountain sickness, of immediate accommodation of the organism to high altitude, and of its long-term acclimatation. In his "Pression Barométrique" of 1878, he established the foundations of high-altitude physiology. In particular, he formulated the «law of partial pressure», the partial pressure of the atmospheric gases alone being responsible for their action on the organism. Paul Bert's historic role, for a long time nearly forgotten, has been recalled to memory by the Swiss medical historians E.H.Ackerknecht and N. Mani. Bert's line of investigation has been followed especially by Angelo Mosso of Turin and, more recently, by F. Verzár, A.von Muralt, A. Fleisch and other Swiss physiologists.

\section{ZuSAMMENFASSUNG}

Es ist das Verdienst von Paul Bert, die Erforschung der "Bergkrankheit», der Höhen-Umstellung (Akkommodation) und der längerfristigen Höhen-Anpassung (Akklimatisation) als eine wesentlich experimental-physiologische Aufgabe erkannt zu haben. In seiner "Pression Barométrique» (1878) sind die grundlegenden Lösungsansätze angegeben und die Teilgebiete einer späteren Forschung skizziert. Diese monumentale, über Jahrzehnte nahezu in Vergessenheit geratene Leistung wurde von Medizinhistorikern der Schweiz wieder in das Bewusstsein gerückt. Gleichzeitig besann sich die physiologische Höhenforschung auf ihren durch Bert bestimmten Ausgangspunkt.

It is always helpful to look at living

processes with the eye of a planning

engineer.

Alexander von Muralt (1954) ${ }^{1}$

Wer aus dem Flachland kommt oder von den sanften Erhebungen eines Mittelgebirges, der fühlt sich von dem unmittelbaren Naturerlebnis des 
Hochgebirges überwältigt. Um so mehr, wenn in kurzer Zeit ein Aufstieg in dreitausend Meter Höhe erreicht wird, wie der auf die Forschungsstation des Jungfraujoch (3454 m). Besonders eindrucksvoll, wenn dies unter sachkundigster Führung, wie der von Alexander von Muralt (1903-1990) ${ }^{2}$ stattgefunden hat. Der Verfasser denkt dankbar an seine eigene Exkursion aus Anlass des Physiologenkongresses in Zürich 1961. Er denkt an die beeindruckende Warnung vor dem «Höhenrausch», an die allerorts sichtbare Mahnung «Walk slowly», um die ohnehin beim Unangepassten angespannte Sauerstoffversorgung nicht durch vermehrten Arbeitsaufwand weiter zu gefährden.

\section{I.}

Die Erforschung der «Höhenkrankheit» als eine Aufgabe der experimentellen Physiologie erkannt zu haben, war das Verdienst von Paul Bert. Dieser geniale Forscher hat die Grundlagen für alle späteren Entwicklungen der Höhenphysiologie gelegt. Bis auf den heutigen Tag besteht aber ein frappantes Missverhältnis zwischen der reellen wissenschaftlichen Leistung von Paul Bert und seiner Annahme durch die Geschichtsschreibung.

Das unsachliche Urteil, etwa des Amerikaners Garrison (1914) ${ }^{3}$, würde fortbestehen (die grosse Brockhaus-Enzyklopädie von 1987 kennt nicht einmal das Stichwort Paul Bert), hätten nicht zwei Schweizer Medizinhistoriker - die einzigen weltweit - die sinnentsprechende Einordnung von Paul Bert in den Gesamtbereich der Wissenschafts- und Kulturgeschichte vollzogen: Erwin H.Ackerknecht und Nikolaus Mani. 1966 schrieb Mani im Gesnerus $^{4}$ :

«Sein Hauptwerk erreicht durchaus das Niveau seines Meisters Claude Bernard.»

Was dies bedeutet, wird erst klar, wenn man bedenkt, mit welchen Superlativen die Geschichte Claude Bernard ${ }^{5}$ bedacht hat.

Wir wissen von Paul Bert ${ }^{6}$, dass er 1833 in Auxerre (Yonne) geboren wurde, sich durch hervorragende Schulleistungen, vorzüglich in Mathematik, ausgezeichnet hat, vom Eintritt in die Ecole polytechnique vom Vater abgehalten, zunächst ohne Begeisterung Rechtswissenschaft studierte, zwar den Doktorgrad erwarb, aber seine Einstellung zur Jurisprudenz mit den Worten kennzeichnete:

«Je critiquais les lois au lieu de les apprendre.» 
Das lässt vermuten, dass das Verständnis für Naturgesetze ihm näher liegen musste als die Beschäftigung mit wandelbaren Satzungen. Das primäre Interesse an den Naturwissenschaften konnte sich in dem anschliessenden Zweitstudium der Medizin in Paris bestätigen.

Zwei Lehrer übten entscheidenden Einfluss auf sein Wissenschaftsverständnis aus, wie er im Vorwort zu seiner Physiologie comparée de la respiration $(1870)^{7}$ bekennt:

«Elève de deux maîtres, Gratiolet et Claude Bernard, auxquels je rends un égal hommage d'admiration, d'affection et de respect, j'ai eu cette heureuse fortune d'être habitué par eux successivement à considérer les faits naturels de deux points de vue différents: le point de vue spéculatif, le point de vue expérimental. Le premier de ces maîtres m'a montré en action les types divers du règne animal; avec le second j'ai commencé à connaître ce que l'homme, disposant de certaines conditions intérieures ou extérieures à un animal donné, peut, les faisant varier à son gré, apporter de troubles instructifs dans l'expression complexe de cette harmonie vivante. Tous deux enseignent que, sous ses formes infiniment variées, le problème de la vie est un: mais l'un cherchant sa solution dans l'analyse des diversités spécifiques, véritables expériences toutes faites que présente la nature, l'autre dans la recherche des conséquences que l'expérience faite au laboratoire entraîne dans l'équilibre d'un seul et même être. L'un remontant des résultats qu'il constate aux conditions qu'il ne peut diriger, et qu'il cherche seulement à connaître; l'autre introduisant à son gré des conditions diverses, pour déterminer leur influence et suivre leurs résultats. En un mot: l'un naturaliste, l'autre expérimentateur: physiologistes tous les deux.»

Bezeichnend ist die Betonung der Eigenständigkeit der Physiologie, die ihn nicht unbedingt die Sympathie der Académie de Médecine gewinnen liess:

«Le but de la physiologie n'est pas seulement d'apporter un simple secours, si important qu'on le suppose, à la médecine. Il est plus élevé. Ce que la physiologie veut connaître, ce sont les conditions de l'équilibre, pour la matière et pour la force, d'un organisme vivant, quel qu'il soit, à quelque degré de l'échelle il se place. A choisir l'homme comme type, les avantages sont innombrables, sous la condition de ne pas se perdre dans la recherche étroite des applications pratiques $[\ldots])^{8}$.

II.

Die von den Genfern Trembley und Charles Bonnet unternommenen Transplantationsversuche ${ }^{9}$ an niedrigen Organismen wurden von Paul Bert auf das Niveau des Warmblüters übertragen ${ }^{10}$, der damit zum Begründer der modernen Transplantationsphysiologie wurde. Sein Interesse konzentrierte sich aber sehr bald auf die Physiologie der Atmung unter dem Einfluss veränderter innerer und äusserer Bedingungen. Neben zahlreichen Einzelar- 
beiten, deren Titel Albert Dastre 1897 im Dictionnaire de Physiologie von Richet ${ }^{11}$ zusammengestellt hat, sind aus diesem Forschungsbereich drei Monographien hervorgegangen ${ }^{12}$.

Bereits in der Physiologie comparée de la respiration von 1870 entwickelt Paul Bert seine künftige Forschungsstrategie. Es geht um den Einfluss des atmosphärischen Drucks auf die Menge der vom Blut transportierten Gase und um die Tatsache, dass dieser Transport komplizierteren Regeln folgt, als sie die Anwendung des Daltonschen Gesetzes vorsieht: «Il y a intervention de quelque affinité chimique», so lautete die Vermutung von FrançoisAchille Longet (1811-1871) ${ }^{13}$.

Die Erfahrungen des französischen Arztes Denis Jourdanet (1815-1891) in grossen Höhen des mittel- und südamerikanischen Kontinents ${ }^{14}$ bestärken Paul Bert in der Annahme einer in verdünnter Luft druckabhängigen, dissoziablen Bindung des Sauerstoffs an das Hämoglobin («hémocristalline»). Gleichsam im Mittelpunkt der von Jourdanet aufgeworfenen Fragen steht die Suche nach den Ursachen für die Symptome der Höhenkrankheit und die Anpassung an erniedrigten Sauerstoffgehalt der Luft bei Dauerbewohnern grosser Höhen, wie in den Anden oder im Himalaya. Es müssten demnach Analysen des arteriellen Blutes durchgeführt werden bei Versuchstieren unter Normalbedingungen, sowie in einem gleichmässigen Luftstrom genau definierter, konstant gehaltener Drucke bei Wirkungsdauern von Minuten bis zu mehreren Tagen. Desgleichen müsste die Antwort auf veränderte Drucke bei demselben Tier untersucht werden.

Erste Versuche mit einer modifizierten Narkosemaske von Claude Bernard waren angelaufen. Der Strassburger Physiologe Piere Dejours ${ }^{15}$ hat auf - bereits vor 1900 von Julius Bernstein (1839-1917) ${ }^{16}$ hervorgehobene Bertsche Versuche zur Messung der inneren, d.h. der Gewebs-Atmung aufmerksam gemacht. Die Einbeziehung des Menschen in Respirationsversuche unter veränderten Drucken war ein Wunschziel, dessen Verwirklichung an der Finanzlage französischer Laboratorien gescheitert wäre, hätte nicht Jourdanet mit einer grosszügigen privaten Stiftung den Weg zu ihrer Durchführung geebnet. 1874 konnte Paul Bert in seiner Monographie Recherches expérimentales sur l'influence que les modifications dans la pression barométrique exercent sur les phénomènes de la vie die von ihm erdachte und im Laboratorium der Sorbonne funktionierende apparative Ausrüstung vorstellen und bereits über entscheidende Ergebnisse berichten.

Von den auf sieben Bildtafeln wiedergegebenen Versuchsanordnungen ${ }^{17}$ seien hier nur zwei herausgestellt : (1) Eine Einrichtung für Kleintiere, die es 


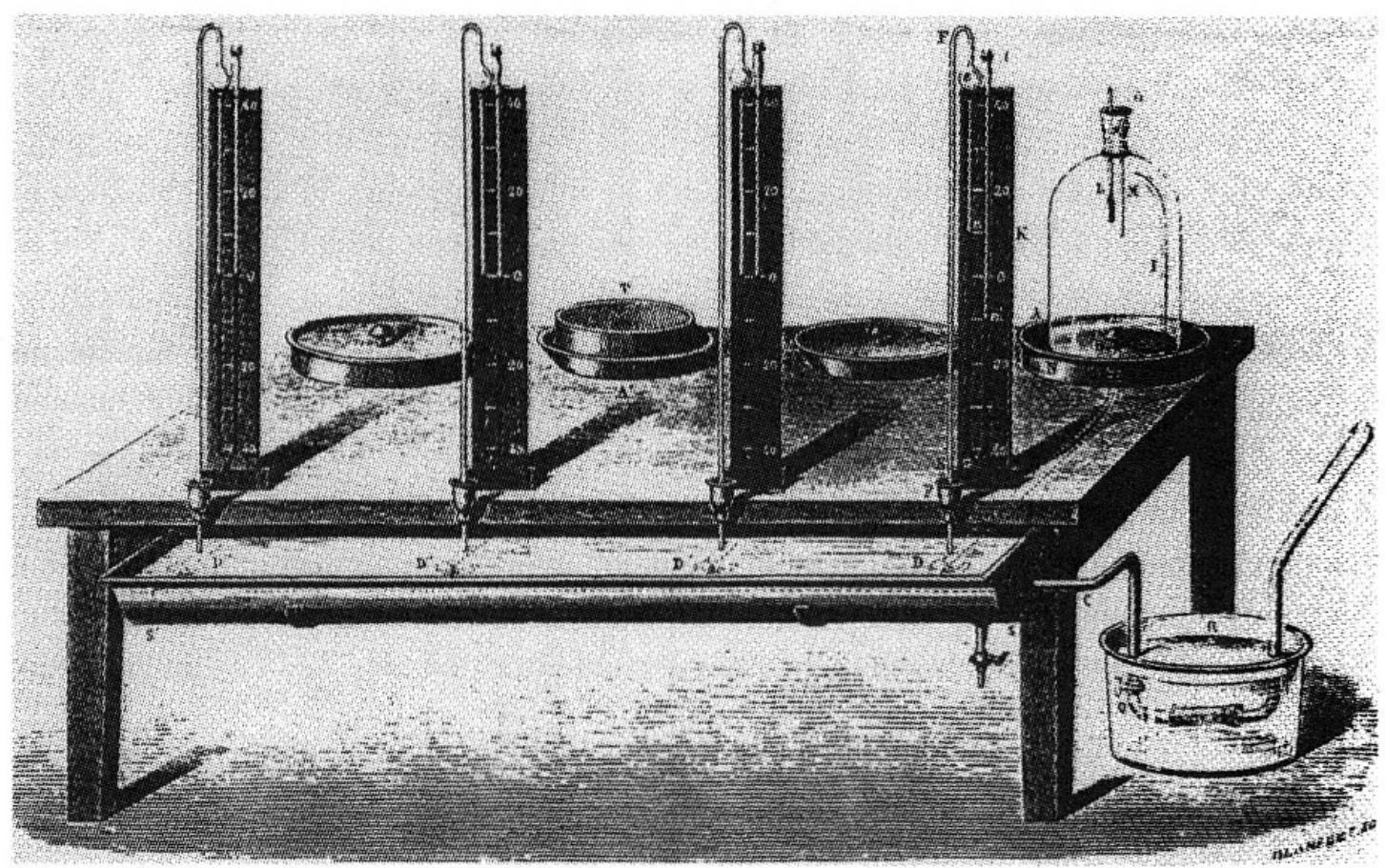

Fig. 1: Appareil pour Expériences simultanées sur la diminution de pression

$A$. Cloche sur plaque avec manchon de zinc $N$, thermomètre $L$, tube de verre pour prendre air $M$, avec robinet noyé dans le manchon plein d'eau 0 . - B. Orifice par lequel la pompe à vapeur aspire l'air, par l'intermédiaire du tube $C$, des robinets $D$ et $Q$ plongés dans l'eau. $-E$ F $G$ H. Manomètre avec communication noyée en $P$. - e. Petit renflement pour éviter les projections de mercure. - $S S$ '. Gouttière de zinc pour noyer les robinets $D D^{\prime} D^{\prime \prime} D^{\prime \prime \prime}$. - T. Appareil à recueillir les urines.

erlaubte, unter gleichen Bedingungen gleichzeitig vier Versuchstiere einer kontrollierten Hypoxie auszusetzen. (Fig. 1), und (2) die Konstruktion (in Differentialanordnung) einer Unterdruckkammer für Respirationsversuche am Menschen (Fig. 2).

Der maschinelle Aufwand für graduierte Kompression und Dekompression sowie die subtilen Massnahmen für die Dichtung der Systeme und die Einbringung möglichst genauer Messeinrichtungen verdienen besondere Beachtung. Paul Bert hatte mit dem Grossversuch am Menschen eine neue Dimension des Experimentierens erschlossen und so das erste moderne Laboratorium für Humanphysiologie geschaffen. Noch nach der Jahrhundertwende benutzte Nathan Zuntz (1847-1920) in seinem Forschungslabor am israelitischen Krankenhaus in Berlin ${ }^{18}$ das von ihm so genannte "pneumatische Kabinett», eine Nachbildung von Paul Berts «grand appareil pour l'étude des faibles pressions» (1874). 
Neben einem ausgiebigen politisch-sozialen und erzieherischen Engagement, das Ackerknecht ${ }^{19}$ und $\mathrm{Mani}^{20}$ ausführlicher gewürdigt haben - Bert war Abgeordneter, Unterrichtsminister unter Gambetta und starb als Statthalter Frankreichs in Indochina - blieb er weiterhin der Wissenschaft verpflichtet. Lediglich das Lehramt, als Nachfolger von Claude Bernard an der Sorbonne, gab er auf. So konnte er 1878 mit über 1100 Seiten, «bis ins feinste Detail ausgearbeitet» (Mani), sein experimental-physiologisches Monumentalwerk La Pression barométrique vorlegen, das ihm 1882 die Pforten der Académie des Sciences öffnete.

Die Pression barométrique besteht aus einem historischen, dem eigentlichen experimentellen und einem kurzen, abschliessenden Teil, der die Schlussfolgerungen enthält. Ackerknecht hat mit Recht auf Berts rund fünfhundert Seiten umfassende, faszinierende Geschichte der Höhenphysio-

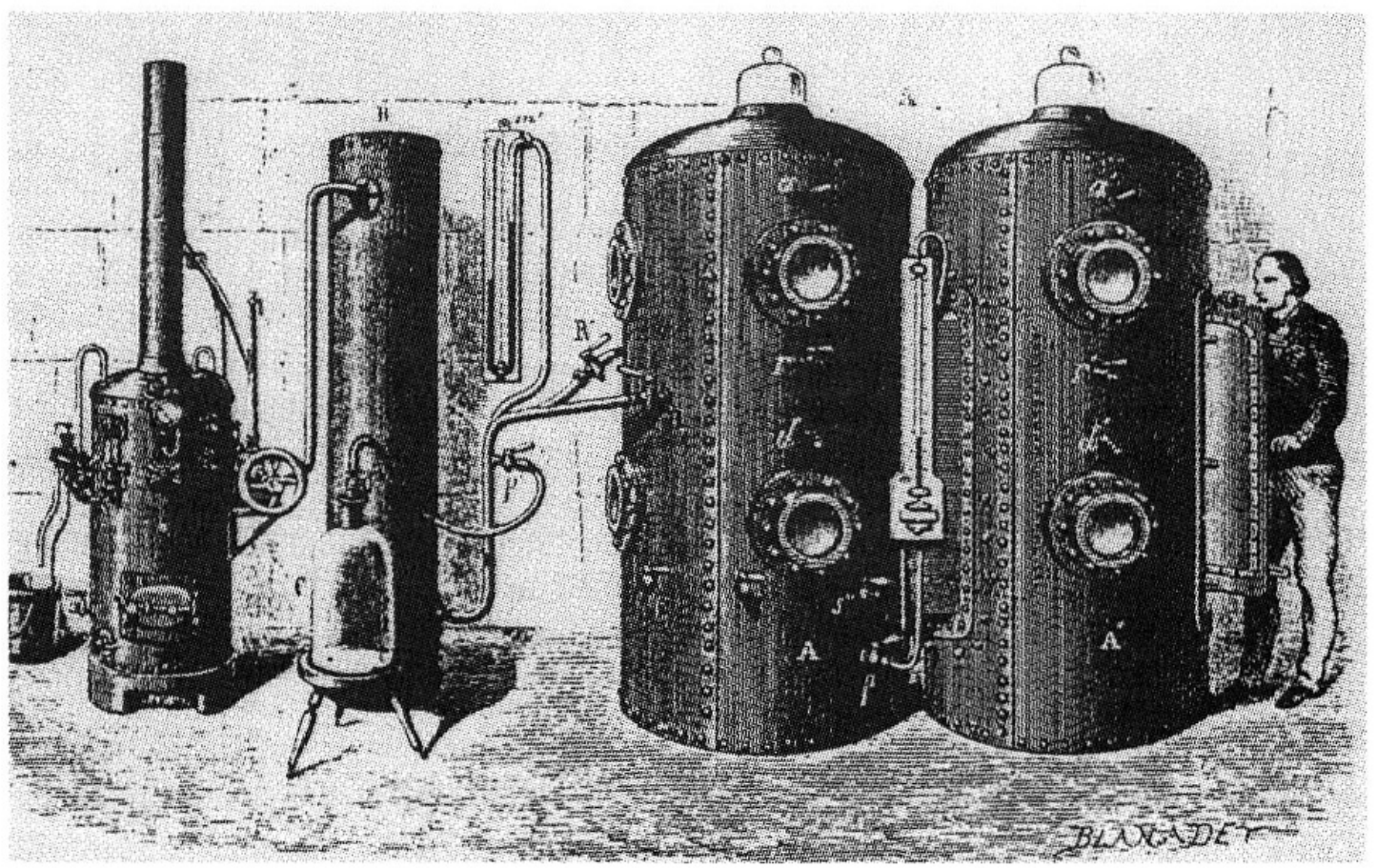

Fig. 2: Grand appareil pour l'étude des faibles pressions

$A, A^{\prime}$. Cylindres en tôle boulonnée, avec hublots en verre. - B. Cylindre où l'on peut faire à l'avance le vide à 7 centimètres, afin d'obtenir une rapide diminution dans les grands cylindres. $-C$. Grande cloche de verre où peut être fait, par l'intermédiaire du cylindre $B$, un vide instantané. $-R, R^{\prime}$. Robinets qui communiquent chacun avec l'un des cylindres $A$ et $A^{\prime}$, que sépare une porte intérieure, marquée en pointillé. - p. robinet de communication avec $C ; \mathrm{r}, \mathrm{r}^{\prime} ; \mathrm{d}, \mathrm{d}^{\prime} ; \mathrm{s}, \mathrm{s}^{\prime}, \mathrm{s}^{\prime \prime}$, ouvertures et robinets pour prendre de l'air des cylindres, extraire le sang, etc. $-\mathrm{a}, \mathrm{a}^{\prime}$. Thermomètres. $-\mathrm{m}, \mathrm{m}^{\prime}$. Manomètres. 


\section{RECHERCHES EXPERIMENTALES \\ SUR L'TRLUENCE QUE LES NODIFICATIONS \\ DANS 1,1 \\ PRESSION BAROMETRIQUE

\author{
EXERCENT SUR LES PHENOMENES DE LA VIE
}

PAB

M. PAUL BERT

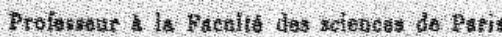

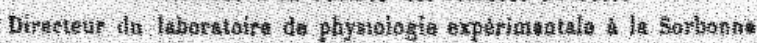

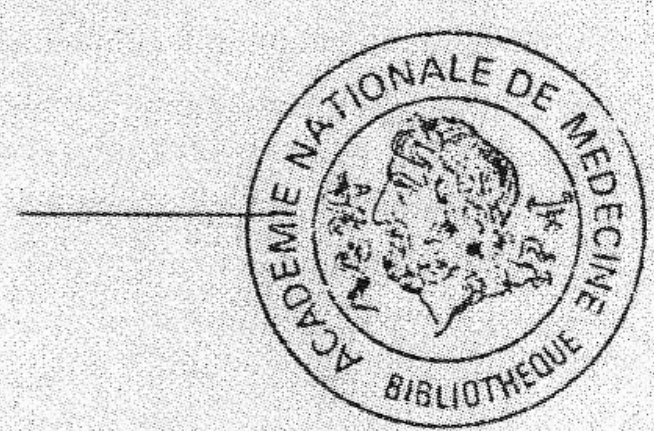

\section{PARIS}

G. MASSON, ÉDITEUR

HBRAIRE DE L'ACADENTE DE MÉDECINE

PLACE oE $\mathrm{L}^{\prime}$ ECOLE-DE-NÉDECINE:

Fig. 3 
logie hingewiesen, die mit der Eroberung der südamerikanischen Bergwelt beginnt, einen Schwerpunkt für die Alpen setzt, die Ballonaufstiege einbezieht und breiten Raum früherer Experimentalforschung und theoretischer Deutung widmet.

Paul Bert bekennt in seinem Vorwort:

«J'ai apporté à la rédaction de l'historique les soins les plus minutieux. Je me suis efforcé de réunir tout ce qui a été écrit sur le sujet de mes études. Il m'a semblé qu'il y aurait grand intérêt pour le lecteur à avoir ainsi sous les yeux toutes les pièces du procès, avec l'infinie variété des récits, leurs contradictions fréquentes, et souvent aussi leur répétitions instructives. J'ai cru devoir prendre le parti de rapporter textuellement les paroles des auteurs cités: je me suis défié des analyses même les plus consciencieuses; il m'est arrivé de voir plusieurs fois, dans mes recherches bibliographiques, les affirmations d'un auteur transformées en négations par une série de traductions et d'analyses.»

Der Leser begegnet ausführlichen Auszügen aus Horace-Bénédict de Saussure (1740-1799) und seiner alpinen Erfahrung (Voyage dans les Alpes, 1786-1796) oder interessanten theoretischen Überlegungen zur «Pressio atmosphaerae, ejus in humanum corpus efficacia» im 3. Band der Elementa Physiologiae corporis humani (1766) von Albrecht von Haller, um aus der Fülle nur zwei Autoren des 18. Jahrhunderts herauszugreifen. Die wörtlichen Zitate stehen immer in einem verbindenden, oft kritisch kommentierenden Text.

Ausgehend von einem Wust widersprüchlicher Hypothesen über die Ursachen der Höhenkrankheit, unter denen allein Jourdanet mit der Annahme einer «Anoxhémie» ein wissenschaftlich tragfähiges Konzept aufwies, so Paul Bert, musste man sich eine Reihe von Fragen vorlegen: Auf welche Weise wirken Änderungen des Luftdrucks auf den Organismus? Welche sind die noch tolerierten Extremwerte? Wie reagiert der Organismus auf die Druckerniedrigung in grossen Höhen, wie auf Druckerhöhung und nachfolgende plötzliche Dekompression? Wie verhält sich das innere Milieu des Blutes unter der Einwirkung erhöhter oder erniedrigter Drucke? Welcher physiologische oder pathophysiologische Mechanismus liegt der Bergkrankheit zugrunde?

Aus den Bertschen Arbeiten lassen sich zwei elementare Gesetze ablei$\operatorname{ten}^{21}: 1$. Das, was Albert Dastre «principe de la modération physiologique» nannte:

«La vertu des choses ne réside pas dans leur nature propre, mais dans leur proportion.»

Ein Übermass an Sauerstoff (Oxydose) ist ebenso schädlich wie dessen Mangel (Anoxie). 2. Das Gesetz der Partialdrucke. Die Wirkung der Gase (und 
des Wasserdampfs) auf das Lebewesen hängt lediglich von ihrem Partialdruck ab, d.h. von ihrer absoluten Menge.

Nikolaus Mani hat in seiner Arbeit von 1966 die Ergebnisse der Pression barométrique nach ihren aufeinanderfolgenden Erkenntnisschritten knapp und übersichtlich aufgeschlüsselt : Mit sinkendem Partialdruck nimmt der Sauerstoffgehalt des Blutes ab. Der Grund ist die leichtere Lösung der Hämoglobin-Sauerstoffbindung. Umgekehrt führt eine Steigerung über den Normaldruck hinaus nicht zu einer weiteren Zunahme des Oxyhämoglobins (Sättigungskurve); nur der physikalisch vom Serum aufgenommene Sauerstoff folgt dem Daltonschen Gesetz. Die arteriovenöse Sauerstoffdifferenz bleibt auch bei sinkendem äusserem Druck lange konstant. Erst ab einer kritischen Schwelle (6 Vol \% $\mathrm{O}_{2}$ ) wird die Differenz kleiner; die Gewebe ersticken.

Die Simulation der Höhenkrankheit im Tierversuch zeigt eine Zunahme von Atem- und Pulsfrequenz, Muskelschwäche, sinkendes Reaktionsvermögen, erlöschende Sensibilität, sinkende Temperatur. Sauerstoffzufuhr ermöglicht, auch bei stark erniedrigtem Luftdruck, das Überleben.

Berühmt wurde der Selbstversuch Paul Berts in der Unterdruckkammer, den man als «Erstbesteigung des Mount Everest» wertete. Bei Luftdrucksenkung auf etwa die Hälfte der Norm $(400 \mathrm{~mm} \mathrm{Hg})$ notierte er: Pulsbeschleunigung, Schwindelgefühl, Übelkeit, Kopfschmerzen, intellektuelle Trägheit, Verdunkelung des Gesichtsfeldes. Diese Beschwerden verschwanden unmittelbar nach Einatmen von Sauerstoff. Bei kontinuierlicher Sauerstoffzufuhr liessen sich Druckerniedrigungen, die einer Höhe von mehr als $8000 \mathrm{~m}$ entsprachen, beschwerdefrei ertragen. Das Maximum der so erreichten Dekompression betrug $250 \mathrm{~mm}=8840 \mathrm{~m}$ Höhe ${ }^{22}$.

«Bert zeichnete in bewundernswerter Weise das Gesamtbild der Höhenkrankheit : Sinkt der Teildruck des Sauerstoffs, so entsteht die Höhenkrankheit des Aeronauten, die Bergkrankheit des Alpinisten, die Beschwerden des experimentell hervorgerufenen Unterdrucks» (Mani).

Trotz einer Fülle grundlegender Erkenntnisse und dem Auffinden eines allgemeinen Naturgesetzes blieb Paul Bert in der physiologischen Forschungsliteratur und sogar in den Lehrbüchern der Physiologie nur wenige Jahre wirklich präsent. Für den ohnehin für Wissenschaftsgeschichte nur wenig aufgeschlossenen reinen Historiker hatte das Erscheinungsbild des Politikers schnell den Forscher verdrängt. Dem Politiker hatte die Nachwelt, in einer wiederum schnell vergessenen Phase, ein prächtiges Denkmal auf der Yonne-Brücke in Auxerre gestiftet und viele französische Ortschaften 
mit einer Rue Paul Bert bedacht, ohne dass man heute recht weiss, wem zu Ehren und warum dies geschah. Benennungen, wie die des «Lycée Paul Bert» («Jacques Amyot») in Auxerre wurden sogar wieder rückgängig gemacht ${ }^{23}$. Dass die Physiologie Paul Bert rasch aus dem Blickfeld verlor, wird offenkundig, wenn man beispielsweise die Geschichte ihrer internationalen Kongresse ${ }^{24}$ durchblättert. Auch der Grund dieses Vergessens wird verständlich.

Der erste dieser Kongresse versammelte 1889, drei Jahre nach dem Tod von Paul Bert, der keine eigentlichen Schüler hinterliess, Physiologen verschiedener Nationen in der "Safranzunft» in Basel. Bereits auf diesem Kongress trat eine Interessengruppe zusammen, deren Ziel die Einbringung des thematischen Schwerpunktes «Höhenphysiologie» für die kommenden Tagungen und eine grosszügige Forschungsplanung war. Organisatoren der ersten Stunde waren Hugo Kronecker (1839-1914, seit 1885 in Bern), der Berliner Nathan Zuntz (1847-1920) und Angelo Mosso (1846-1910) aus Turin, ein Schüler von Moritz Schiff, Carl Ludwig, Claude Bernard, Ranvier und Marey. Die Tagungen in Bern (1895), Turin (1898), Brüssel (1904) brachten eine Fülle neuer Daten und Berichte über die alpine physiologische Forschungsstation auf dem Monte Rosa, die einem internationalen Komitee unterstellt wurde und die Physiologen von Rang Forschungsaufenthalte ermöglichte.

Mosso hatte der gewissermassen «abstrakten» Physiologie der Unterdruckkammern, deren Notwendigkeit aber nach wie vor besteht, die reale hochalpine Bergerfahrung entgegengesetzt. Vor allem war er der Begründer einer neuen Theorie der Bergkrankheit, die er auf ein Kohlensäuredefizit im Blut («Akapnie», besser Hypokapnie) beim Einatmen verdünnter Luft zurückführte. Die Idee der Akapnie war stark genug, in vieler Augen einen «Paradigmawechsel» im Sinne von Kuhn herbeizuführen und so die Bedeutung der Anoxaemie in den Hintergrund treten zu lassen.

Parallel zu Einzeluntersuchungen verschiedener Forscher (darunter Tierversuche an Frosch und Katze), hatte Mosso mit einer Gruppe von Versuchspersonen aus dem italienischen Militär, während des Aufstiegs auf den Monte Rosa und nach deren Stationierung in Höhe des Forschungslaboratoriums, das Verhalten von vorwiegend vegetativen Funktionen untersucht. Getestet wurden neben Registrierung der Atemtätigkeit: der Blutdruck, die Pulsfrequenz, ergänzt durch Messungen mit dem Sphygmomanometer (eine aus den Verfahren von Marey abgeleitete Kombination von Druck- und Volumenmessung am Vorderarm), die Muskeldurchblutung (Myosphygmographie), 
die Ermüdung des Skeletmuskels (aufgezeichnet mit dem von Mosso konstruierten Ergographen), die Ermüdung des Herzmuskels, Störungen der Temperaturregulation, der Verdauungstätigkeit, der nervösen Erregbarkeit. Wie bei Paul Bert musste Versuchen über den Chemismus der Atmung, an denen auch die wissenschaftlichen Gäste N. Zuntz und A. Loewy teilnahmen, ein besonderes Gewicht zukommen.

Als bedeutsamer Faktor für das Auftreten der Bergkrankheit erwies sich nicht der Sauerstoffmangel auf allgemeinem Gewebsniveau, sondern die verringerte $\mathrm{CO}_{2}$-Spannung des Blutes. Dies veranlasste Mosso zur Aufstellung seiner neuen Doktrin. Die Mehratmung unter Dekompression führt zu fortschreitender Eliminierung der Kohlensäure und damit zum Kohlensäuredefizit im Blut (Akapnie). Einatmung eines Sauerstoff-Kohlensäuregemisches beseitigt die Höhensymptome:

«L'accumularsi dell'acido carbonico nel sangue è un excitamento specifico più forte per il centro respiratorio, che non sia la mancanza dell'ossigeno» ${ }^{29}$ (S.400).

Die vielfach vermutete Verringerung der Durchblutung des zentralen Nervensystems als mögliche Ursache der Höhenkrankheit liess sich nicht bestätigen :

«Nessuno che abbia pratica di simili studi sulla circolazione del cervello, oserebbe affermare che i mutamenti osservati nel polso cerebrale (leichte Abnahme der Amplitude) possono essere la causa del malessere prodottosi. La conclusione è questa, che le modificazioni caratteristiche osservate nella sensibilità, nell'intelligenza e nel moto, di quanti fecero delle ascensioni aerostatiche, o sui monti, non possono spiegarsi con l'esistenza di una anemia, o di una congestione del cervello. Il sangue circola in quantità sufficiente nel cranio e in modo poco diverso dal normale fino all'altezza di 5520 metri» (Einatmung eines künstlichen Mangelgemisches, ${ }^{29}$ Cap. 18).

Eine «mechanische» Theorie der Druckeinwirkung auf das Herz stellte Kronecker auf, gefolgt von dem Brüsseler Physiologen Paul Heger (18461925). 1903 hatte Bartlett vom Physiologischen Institut Bern im American Journal of Physiology über Änderungen des Blutdrucks beim Atmen verdünnter Luft berichtet ${ }^{25}$. Im gleichen Jahr war auch Kroneckers Monographie Die Bergkrankheit ${ }^{26}$ erschienen. Nach Kronecker hat die Bergkrankheit ihre Ursache weder im Sauerstoff- noch im Kohlensäuremangel, sondern in mechanischen Störungen des Lungenkreislaufs mit Wirkungen wie bei Insuffizienz der Mitralklappe ${ }^{27}$. Bemerkenswerterweise behauptet bei Léon Binet (1891-1971), in dessen Handbuch (1934) ${ }^{28}$, diese Theorie einen gleichwertigen Rang neben «Hypoxaemie» und «Akapnie». Doch fesselte die Akapnie in ungleich stärkerem Masse das Interesse. 
1898, zwanzig Jahre nach Berts «Pression barométrique», war bereits in zweiter Auflage die brillant geschriebene, neben dem Werk von Paul Bert mit noch nicht fünfhundert Seiten überaus handliche Fisiologia dell'Uomo sulle Alpi von Angelo Mosso ${ }^{29}$ erschienen. Mosso würdigt darin die Persönlichkeit von Paul Bert

«col suo talento meccanico, coi suoi apparecchi e le analisi del sangue $[\ldots]$ che gettò i primi fondamenti di una fisiologia dell'uomo sulle Alpi»,

kommt aber bald zu dem Schluss:

«La vastità dell'opera scientifica alla quale P. Bert pose mano, fu superiore alle sue forze. Noi non dobbiamo meravigliarci se altri venuti dopo lui rettificarono i risultati di alcune suoi indagini.»

Mosso zitiert die Berliner Schule. Die Arbeiten über den Blutchemismus von Christian Bohr, August Krogh, mit immer präziseren Daten, taten das ihre, um die Anfänge vergessen zu lassen. Der Experimentalforscher verzichtet auf aufhaltsame Rückblicke. Ich erinnere mich an einen Ausspruch von Alexander von Muralt in der Bibliothek des Hallerianum. Etwa sinngemäss :

Physiologische Publikationen, älter als fünf Jahre, kommen bei uns in die Reserve. Sie haben nur geringen aktuellen Wert.

Den Einfluss der Hypokapnie, aber auch der Hypoxaemie glaubte bereits Kronecker widerlegt zu haben. Doch lässt nach Ludimar Hermann ${ }^{30}$, ganz im Gegensatz zur Einschätzung durch Léon Binet, dessen monographische Darstellung «eine fest begründete Theorie nicht erkennen». Der «Entwertung» der Bertschen Hypoxaemie-Doktrin durch Mossos Theorie der Akapnie wurde später von den angelsächsischen Forschern Douglas, Haldane und Yandell Henderson mit experimenteller Begründung widersprochen. Heute ergibt sich das Bild eines weit komplizierteren Wirkungsgefüges, das aus dem Zusammenspiel verschiedener Faktoren resultiert (Dejours) ${ }^{31}$.

Dem Hauptwerk von Paul Bert ist trotz der - mit Ausnahmen wie Hans Winterstein ${ }^{32}$ oder Yandell Henderson ${ }^{33}$ - sich ahistorisch gebenden Physiologie, ein in ihrer Geschichte wohl einzigartiges Schicksal zuteil geworden: fünfundsechzig Jahre nach ihrem Erscheinen, neunundfünfzig Jahre nach dem Tod des Verfassers, erschien 1943 in den USA, in englischer Sprache mit einer Einführung von John Farquar Fulton, der integrale Text der Pression barométrique $^{34}$. In der Tatsache, dass diese vollständige Übersetzung nicht aus historischen, sondern aus praktischen Gründen im Hinblick auf Fragen des Alpinismus und der militärischen Luftfahrt erfolgte, d.h. nicht der erinnernden Rückschau, sondern der Vermehrung des positiven Wissens 
diente, darin sieht Ackerknecht ${ }^{35}$ einen "Triumph», wie ihn kein anderer Physiologe des 19. Jahrhunderts erlebt hat. Er kommt zu dem Schluss: Paul Bert schrieb nicht nur Geschichte als der «richtige Mann im richtigen Geist», die Aufzeichnung und Besprechung seiner 668 eigenen Versuche hätte der späteren Forschung manchen Irrtum und manche unnütze Wiederholung erspart, wenn sie sie nur gekannt hätte. So «entdeckten» manche Neuen, was Paul Bert lange vor ihnen entdeckt hatte.

Die Gliederung des Forschungsprogramms in physiologische Teilgebiete, von Atmung und Kreislauf bis zum Kohlenhydratstoffwechsel, dem Wärmehaushalt, der Harnsekretion (im 3. Kapitel des Experimentalteils) zeigt, woran sich künftige Bemühungen anschliessen konnten.

\section{III.}

In der Schweiz wurden in den vierziger Jahren, zur Zeit der Wiedererwekkung von Paul Bert, Höhenklimaforschungen durchgeführt, die in zwei zweiteiligen Veröffentlichungen, 1944-1948, zusammengefasst sind ${ }^{36}$. Die Expeditionen auf das Jungfraujoch und die Laborarbeiten der Forschungsstation wurden von Alexander von Muralt geleitet und von Alfred Fleisch, dem der Verfasser seine erste Ermutigung in der Physiologie verdankte, sowie von Fritz Verzár, der Arbeiten des seit Friedrich Miescher (1844-1895) in der Höhenforschung aktiven Basler Instituts vorstellte.

Neu ist die stärkere Einbeziehung der animalischen Physiologie. Erstmalig sind Untersuchungen an einer grösseren Zahl von Versuchspersonen, wie schon von Mosso mit seinen Soldaten angestrebt, unter streng kontrollierten Ausgangsbedingungen durchgeführt, vergleichsweise in der Höhenlage von Lauterbrunnen (800 m) und auf dem Jungfraujoch (3457 m). Die veränderten Bedingungen wirken als eine Summe von Reizen, wobei die Minderung des Sauerstoff-Partialdrucks der wirksamste Faktor ist.

Im Rahmen des klassischen Schemas «Umstellung (Akkommodation) und Anpassung (Akklimatisation)» werden die Reize nach Dauer, Stärke, Verteilung und Rezeptorempfindlichkeit differenziert. Neue, verfeinerte Messmethoden, wie etwa für die Kreislaufphysiologie in Fribourg unter Aloys Müller entwickelt (Pulsregistrierung), konnten hier zur Anwendung kommen. Zu ihrer Prüfung ist der starke Höhenklimareiz ein besonders brauchbares Kriterium. Die Auswertung bediente sich der von Fueter für die Dispersion (mittlerer Fehler) angegebenen Regeln. 
Allgemeine Arbeitshypothese war, nach den neuen Konzeptionen von Walter Rudolf Hess, die Annahme einer Veränderung des vegetativen Gleichgewichts durch den Höhenreiz. Die Auswirkung konnte durch Messung der Schwellen des Druck- und Geschmacksinns, der Pulszeiten, der Pulswellengeschwindigkeit (Zunahme durch Änderung des Elastizitätsmoduls der Gefässe), der zirkulierenden Plasmamenge, der Magensaftsekretion, des Blutzuckerspiegels erfasst werden.

Zur Stabilisierung der Messwerte wurden die Versuchspersonen für eine zwischen drei und sechs Tage dauernde «Vorperiode» im Hotel in Lauterbrunnen «interniert» bei absolutem Rauch-, Alkohol- und Kaffeeverbot, mässiger Bewegung, gemeinsamer Diät und rechtzeitiger Nachtruhe. Durch eine grosse Anzahl von Messpunkten wurden praktisch «ungebrochene» Messkurven erhalten. Dem Höhenaufenthalt auf dem Jungfraujoch (acht bis zwölf Tage) folgte eine zweitägige Nachbeobachtung zur Erfassung des «Abstiegseffekts». Aus dieser Versuchsorganisation wird deutlich, dass gegenüber allen früheren Forschungen ein völlig neuer Weg eingeschlagen wurde.

Bemerkenswert ist das Ergebnis der «Amphotonie» durch Höhenreiz, d.h. einer gleichzeitigen Tonussteigerung im sympathischen und parasympathischen System mit Überwiegen des Orthosympathicus. Diese Amphotonie schwindet mit fortschreitender Akklimatisation. Bei künstlichen Höhen scheint die Vagotonie zu überwiegen. Die von Fleisch und Grandjean entdeckte Reizschwellensenkung im nervösen Gesamtsystem wird als typische Wirkung des natürlichen Höhenreizes erkannt. Da sie nach dem in den Bergen verunglückten Erich Opitz (Kiel) auf Hypoxie $\left(\mathrm{O}_{2}\right.$-Mangel-Depolarisation der Rezeptormembranen) beruhen soll, müsste sie auch im Druckkammerversuch nachweisbar sein.

Auf das besterforschte Objekt, das Blut, konzentrierte sich, schon seit Miescher, das Basler Institut. Daneben stand die Erforschung der Atmung (Abnahme der Vitalkapazität), insbesondere der Aerosolwirkung der Luft (Kondensationskerne), der inneren Sekretion und der Wärmeregulation im Vordergrund.

Für die vermutete erhöhte Sauerstoff-Transportkapazität des Blutes bei Höhenanpassung könnte man, Paul Bert folgend, eine qualitative Änderung des Hämoglobin, eine Hämoglobinzunahme im einzelnen Erythrocyten, oder ein Anwachsen der Erythrocytenzahl verantwortlich machen. Paul Bert hielt eine Höhen-Erythrocytose für das Wahrscheinlichste. Sie wurde alsbald, am Anfang der neunziger Jahre von François Viault, Anatom und 
Physiologe aus Bordeaux, in den Anden nachgewiesen. Mani ${ }^{37}$ sieht in dieser revolutionierenden Feststellung, dass eine als konstant angesehene biologische Grösse (die «normale» Erythrocytenzahl) sich durch den Höhenreiz spektakulär verändert, die Hauptanregung für den Beginn einer systematischen Höhenforschung, wie sie anschliessend Kronecker, Mosso, Adolf Loewy und andere betrieben.

Ausgehend von der These, dass immer das Zerfallsprodukt der Reiz für die Neubildung ist, lassen sich unter dem Einfluss der Höhe zwei parallel zueinander verlaufende Prozesse nachweisen: eine vermehrte Zerstörung alter und eine vermehrte Entstehung hämoglobinreicher neuer Erythrocyten. Die allgemeine Zunahme des Erythrocytendurchmessers (Volumenvergrösserung) führt zu Riesenformen, die rasch zerfallen. Aus den über Jahrzehnte durchgeführten Untersuchungen geht nach Verzár hervor:

«Der Sauerstoffmangel in grosser Höhe, auf $3450 \mathrm{~m}$ ü. M., mit nur $85 \% \mathrm{O}_{2}$ im arteriellen Blut, verursacht ein Anschwellen der Erythrocyten, was zu einer vermehrten Hämolyse führt. Das freiwerdende Hämoglobin wird zu Bilirubin (Höhen-Bilirubinämie) und dieses (vielleicht auch andere Zerfallsprodukte der Erythrocyten) sind die Reize der vermehrten Neubildung.»

Es ergaben sich annähernd fünfzig differenzierte Versuchsprogramme, zu deren Durchführung sich die Spitzenforscher schweizerischer Hochschulen vereinigten. Liest man diese von v. Muralt, Fleisch und Verzár herausgegebenen Berichte nach, dann findet sich am Beginn ihrer Übersicht, wie selbstverständlich, der Name von Paul Bert. Und dahinter steht sein, als Bertsches Gesetz in die Naturwissenschaft eingegangener, Leitgedanke:

«La diminution de la pression barométrique n'agit sur les êtres vivants qu'en diminuant la tension de l'oxygène dans l'air qu'ils respirent.» 


\section{Anmerkungen}

1 A. v. Muralt: Development and limitation of physiological research, in: Ilza Veith (ed.): Perspectives in Physiology, Washington D. C. 1954, S. 12.

2 «Mit Beginn seiner Tätigkeit als Ordinarius schlug ihn W. R. Hess, der Zürcher Fachkollege und spätere Nobelpreisträger dem Stiftungsrat für die Hochalpine Forschungsstation Jungfraujoch als Nachfolger im Amt des Präsidenten vor.» Robert Stämpfli, Nachruf auf A. v. Muralt, Deutsche Physiologische Gesellschaft, 1991.

3 F. H. Garrison: An introduction to the History of Medicine, Philadelphia/London 1914, S. 495. Dazu Ackerknecht (Anmerkung 19) und G. Rudolph: L'initiateur Paul Bert (18331886) et la physiologie de haute altitude, $116^{\circ}$ Congrès National des Sociétés Savantes, Chambéry 1991, Histoire des sciences et des techniques (im Druck).

4 N. Mani : Paul Bert als Politiker, Pädagoge und Begründer der Höhenphysiologie, Gesnerus 23, 109-116 (1966).

5 G. Rudolph: Claude Bernard (1813-1878) in: D. v. Engelhardt u. F. Hartmann (eds.) Klassiker der Medizin II, München 1991, S. 135-159 u. 411-415.

6 Eine zuverlässige und moderne Biographie schrieb Pierre Mercier: Paul Bert (1833-1886), Bull. Acad. Nat. Méd. 162, 886-898 (1978). Aus Anlass des Centenaire de la Pression Barométrique hat die Bibliothèque der Académie Nationale de Médecine unter Catherine Lupovici, Monique und Jacqueline Chapuis einen reich dokumentierten Katalog und eine, gegenüber früheren biographischen Angaben, revidierte Chronologie erstellt.

7 P. Bert: Leçons sur la Physiologie comparée de la Respiration, Baillière et Fils, Paris 1870, $588 \mathrm{~S}$.

8 P. Bert: Ibid. S. XI; S. auch Mercier: loc. cit., S. 886.

9 G. Rudolph: Les débuts de la transplantation expérimentale - Considérations de Charles Bonnet (1720-1793) sur la «greffe animale», Gesnerus 34, 50-68 (1977).

10 P. Bert: De la greffe animale, Paris 1863 - Ders.: Recherches expérimentales pour servir à l'histoire de la vitalité propre des tissus animaux, Paris 1866.

11 A. Dastre: Paul Bert, in: Charles Richet (ed.) Dictionnaire de Physiologie, T. II, Paris 1897, S. 85-90.

12 P. Bert: Leçons sur la Physiologie comparée (s. Anm. 7) - Ders.: Recherches expérimentales sur l'influence que les modifications de la pression barométrique exercent sur les phénomènes de la vie, Baillière, Paris 1874, 170 S. - Ders.: La Pression barométrique. Recherches de physiologie expérimentale, Masson, Paris 1878, $1168 \mathrm{~S}$.

13 F. A. Longet: Traité de Physiologie I, Masson, Paris 1861, S. 493.

14 D. Jourdanet: Les altitudes de l'Amérique tropicale comparées au niveau des mers au point de vue de la constitution médicale, Baillière, Paris 1861. - Ders.: Du Mexique du point de vue de son influence sur la vie de l'homme, Baillière, Paris 1861.

15 P. Dejours: Paul Bert Commemoration Issue - One hundreth anniversary of the publication of «Leçons sur la physiologie comparée de la respiration» (Introduction), Respiration Physiology 9, Amsterdam 1970, S. V-VIII.

16 J. Bernstein: Lehrbuch der Physiologie des thierischen Organismus ${ }^{2}$, Enke, Stuttgart (1884, 1900), S. 133/134.

17 P. Bert: Recherches expérimentales [...], op. cit. (s. Anm. 12) Pl. 1 u. 3.

18 H. C. Gunga: Leben und Werk des Berliner Physiologen Nathan Zuntz (1847-1920), Mathiesen, Husum 1989. 
19 E. H. Ackerknecht: Paul Bert's Triumph, in: H.E. Sigerist (ed.) Essays in the History of Medicine, Baltimore 1944, S.16-31.

20 N. Mani: Paul Bert [...], loc. cit. (s. Anm.4).

21 A. Dastre: Paul Bert, loc. cit. (s. Anm.11).

22 P. Bert: La Pression barométrique, op. cit. (s. Anm. 12), S. 762.

23 P. Mercier: Paul Bert, loc. cit. (s. Anm.6), S. 887.

24 K. J. Franklin: A short history of the international Congresses of Physiologists 1889 1938, Annals of Science 3, 242-335 (1938) Reprod. facsimile in: Wallace O.Fenn (ed.): History of the International Congresses of Physiological Sciences, Baltimore 1968.

25 Bartlett: On the variations of blood-pressure during the breathing of rarefied air, Amer. J. Physiol. 10, 149-163 (1903).

26 H. Kronecker: Die Bergkrankheit, Urban \& Schwarzenberg, Berlin u. Wien 1903. - Die physikalische Orientierung von Hugo Kronecker wird aus seiner mathematisch-naturwissenschaftlichen Begabung und seinem Schülerverhältnis zu Helmholtz, Wundt und vor allem Carl Ludwig bestimmt. In Bern konnte er der Tradition von Gabriel Gustav Valentin folgen.

27 Vgl. L. Hermann: Jahresbericht über die Fortschritte der Physiologie, Bd. XII (1905).

28 L. Binet: La dépression barométrique - Le mal des altitudes, in: G. H. Roger et L. Binet (dir.): Traité de Physiologie normale et pathologique, T. V. Respiration, Masson, Paris 1934, S. 431-441.

29 A. Mosso: Fisiologia dell'Uomo sulle Alpi, Studi fatti sul Monte Rosa, Nuova edizione, Treves, Milano 1898.

30 L. Hermann: Atembewegungen, in: Jahresbericht [...], loc. cit. (s. Anm. 27), S. 17.

31 P.Dejours: La respiration en haute altitude, in: Ch. Kayser (ed.) Physiologie T. III, Les grandes fonctions, Flammarion, Paris 1963, S. 192-205.

32 H. Winterstein: Die Narkose in ihrer Bedeutung für die allgemeine Physiologie, Springer, Berlin 1926. S. auch die von Yandell Henderson zum Thema «Bergkrankheit und Akklimatisierung» zitierten Arbeiten von Winterstein (1911-1934).

33 Y. Henderson: Adventures in Respiration, Williams \& Wilkins, Baltimore 1938 (deutsch, Leipzig 1941).

34 P. Bert: Barometric Pressure. Researches in Experimental Physiology, Translated into English by M. A. Hitchcock and F. A. Hitchcock, Columbus, Ohio 1943. $32+1055$ S.

35 E. H. Ackerknecht: Paul Bert's Triumph, loc. cit. (s. Anm. 19).

36 A. v. Muralt (ed.) Klimaphysiologische Untersuchungen in der Schweiz, Teil 1, Schwabe, Basel 1944. - A. Fleisch u. A. v. Muralt (eds.) zum Teil 2, Basel 1948. - F. Verzár (ed.): Höhenklima-Forschungen des Basler Instituts (Sir Joseph Barcroft gewidmet), Schwabe, Basel (I) 1945, (II) 1948.

37 N. Mani : Paul Bert in: Dictionary of Scientific Biography 2, New York 1970, S. 59-63.

Prof. Dr. G. Rudolph

(Universität Kiel)

Jamm-Str. 4

D-7630 Lahr 